\title{
Prevalence of Adverse Childhood Events (ACEs) and Its Relationships to Life-Satisfaction Among Patients with Psychiatric Disorders
}

\author{
Wachiraporn Arunothong \\ Department of Psychiatry, Lampang Regional Hospital, Lampang, Thailand
}

\section{Email address:}

wachiraarun@gmail.com

\section{To cite this article:}

Wachiraporn Arunothong. Prevalence of Adverse Childhood Events (ACEs) and Its Relationships to Life-Satisfaction Among Patients with Psychiatric Disorders. American Journal of Pediatrics. Vol. 6, No. 4, 2020, pp. 421-427. doi: 10.11648/j.ajp.20200604.15

Received: September 23, 2020; Accepted: October 7, 2020; Published: October 17, 2020

\begin{abstract}
Objective: To demonstrate the prevalence of ACEs and the association of ACEs' effects on life- satisfaction among patients with psychiatric disorders. Methods: Patients with psychiatric disorders aged $\geq 18$ were enrolled. Patients who had dementia and could not read or write in Thai were excluded. Patients were asked to complete demographic, ACEs, and life-satisfaction questions. The study was held in a psychiatric clinic with prompt intervention to help patients who might be sensitive to ACEs questions. Results: 271 patients, with a mean age of 37.6 years (range 18-80 yr.) completed questionnaires. $66.8 \%$ were males. $59.4 \%$ of patients suffered from substance related disorders. Overall, $45 \%$ of patients had $\geq 1$ ACE and of these, $5.5 \%$ had $\geq 4$ ACEs. The top three ACEs were feelings of abandonment by a family, parents divorced or separated, and being a witness of domestic violence. After adjusted confounders, ACEs could significantly increase feeling bored with life; feelings of failure; and feelings of suffering in life; and significantly decrease feelings of life-satisfaction; feelings of ease and happiness; feeling safe; and feeling love in a family; feeling of accepting; and handling of difficult situations. The protective factors of ACEs were living with both parents, living with either of them, and living in a family that could support each other during difficulties. Conclusion: The prevalence of ACEs among patients with psychiatric disorders is quite common. ACEs showed negative relationships with later life-satisfaction and happiness. The graded negative relationships of ACEs to lifesatisfaction found at every ACE that increases. Solutions to decrease the impacts of ACEs are to promote parents to live with their children during their childhood periods and to strengthen family relationship to support each other during difficulties.
\end{abstract}

Keywords: Adverse Childhood Events, Prevalence, Thailand, Patients with Mental Illness, Life-satisfaction

\section{Introduction}

Adverse childhood event (ACE) is a traumatic event that occurs during childhood periods, before the age of 18 years old. According to the CDC-Kaiser Permanete ACE study [1], the ACEs include childhood abuse, neglect, and living in a dysfunctional family. Childhood abuse includes physical abuse, sexual abuse, and emotional abuse. Neglect includes physical and emotional neglect. Household dysfunction includes parental divorce or separation, living with a family member who has mental illness, living with a family member who is/was in a prison, and being a witness of domestic violence among parents.

Study of the CDC-Kaiser Permanete ACE study [1] demonstrated the strong and graded relationship between
ACEs and chronic illness in both physical and mental illness and health risk behaviors. People who had more ACEs tend to suffer more from those illnesses.

The CDC-Kaiser Permanete ACE study [1] reported that more than $50 \%$ of participants had at least $1 \mathrm{ACE}$ and around $25 \%$ had more than 2 ACEs. Participants who had more than 4 ACEs had 4 to 12 time-higher risks in consuming alcohol, doing illegal drugs, committing suicide, and experiencing depression. In addition, these people had 2 to 4 times higher risk for developing obesity, diabetes, heart disease, cancer, stroke, chronic obstructive pulmonary disease, and sexually transmitted diseases. Also ACEs had shown negative association with educational, job opportunities, and later life satisfaction.

Although the negative impact of ACEs is enormous 
especially the impacts to mental health and health risk behaviors, there were very few studies in Thailand that have reported about the prevalence of ACEs and its negative impacts. In this study only related studies in Thailand were reviewed as the aim of our study is to compare our study to other previous studies in Thailand that have similar objectives in studying ACEs in patients with mental illness and in patients who are involved with substances. Six literatures that relates to this study were reviewed. (1) Isaranurug et al. in 2002 [3] reported that $95.3 \%$ of grade 6 students reported that they had been abused. The most common acts that they received were beaten by cane or belt and scolding. Child's gender, family crises and family relations could be risk factors for household abused by parents. (2) Panyayong B. et al. in 2013 [2] that studied in grade 4 students reported about factors that are significantly associated with behavior and emotional problems for the children included family's factors; family discord, large family with more than 4 children, death of family members in the previous 6 months, children not living with biological parents, and parental mental illness, substance abuse or criminal record, and children's factor was; children who repeated grade at school. (3) Jirapramukpitak T et al. in 2005 [4] did a study in a community with young Thai people whose age 16-25 and found that the prevalence of child abuse was around $38 \%$. The study also reported that common mental disorders significantly associated with emotional abuse, and alcohol use disorders associated with sexual abuse. (4) Panyayong B. et al. in 2013 [5] reported the association of child maltreatment, mental disorders, and re-victimization by intimate partner violence (IPV) in adulthood that $5.3 \%$ of patients with mental health disorders aged over 18 reported about childhood trauma. Women with history of sexual abuse and witnessing domestic violence were likely to have suicidal behaviors whereas men with history of witnessing domestic violence were significantly associated with substance use. Women who reported violence were likely to be revictimized by IPV. Men who witnessed domestic violence were re-victimized by IPV. (5) Htike M. et al. in 2017 [6] that studied the ACEs, anxiety, and depression that predicted the associated factors of methamphetamine use among vocational students reported that $55 \%$ of participants had ACEs. $44.9 \%$ were screened anxiety, $44.0 \%$ seemed to have depression, and $2.4 \%$ used methamphetamine. And (6) Ham E. et al. in 2019 [7] assessed the prevalence of polysubstance use and associated factors among grade 11 students and found that $31.1 \%$ of participants had ACEs. $19.5 \%$ of students were polysubstance users. In addition they reported that physical neglect, and household alcoholism/drug abuse associated with poly substance use and students with high ACEs had more chance to be a polysubstance user.

As reviews above, the previous studies focused on prevalence and impacts only on children, adolescents and young adults. Only one study from Panyayong B. et al. in 2013 [5] demonstrated the prevalence of abuse and the association of abuse to mental illness among adults. However, in Panyayong B. et al (2013) study, only 3 types of abuses were examined; including childhood sexual abuse, childhood physical abuse, and children who have witnessed domestic violence. Other dimensions of abuse such as emotional abuse, neglect, and others dysfunctional family problems, which are also important items of ACEs, were not studied. Therefore, there is a gap in the knowledge of ACEs in adults in Thailand in terms of prevalence and their association to mental health problems and health risk behaviors in adulthood.

Studies also mentioned relationship between adverse events in childhood have affected to adulthoods' happiness and life-satisfaction. [10-14, 18]. Those studies explained that ACEs had significantly affected the sense of social wellbeing and psychological well-being [13, 18]. People who had ACEs tend to have lower educational success $[1,18,20]$; unhealthy behaviors such as using substances $[1,6,7,18]$; violence behaviors [1]; mental illness [1, 4, 5, 18]; poor health-related to quality of life; and shorter life expectancy [1, $18,20]$. In addition, ACEs had strong and graded relationships to neuroticism and emotion-focused coping and lower conscientiousness; lower agreeableness; lower emotional intelligence; and had higher task coping difficulties. These resources were significantly related to increased stressors and lower wellbeing in adulthood [14].

As ACEs associated with mental illness and health risk behaviors including using substances, this study aims to explore both prevalence of ACEs in adults with mental illness and substance abuse and also find the association of ACEs to later life satisfaction.

Objectives

To study the prevalence of ACEs among patients with psychiatric disorders.

To find the association of ACEs and later life-satisfaction among patients with psychiatric disorders.

\section{Methods}

Patients with psychiatric disorders aged $\geq 18$ were enrolled. Patients who had dementia and could not read or write in Thai were excluded. Patients were asked to complete demographic; ACEs; and life-satisfaction questions. The study was held in a psychiatric clinic with promptly intervention to help patients who might be sensitive to ACEs questions. Patients who felt uncomfortable with ACEs questions could withdraw from the study at any time and can ask for help from psychiatric nurses. The study was approved by the institutional review board.

\subsection{Instruments}

The ACEs questions contained 10 events which included all forms of abuses, neglect, and household dysfunctions. Each ACE question was a yes/no question and the score was counted as 1 point when the answer was yes. The minimum score was 0 and the maximum score was 10 . The questionnaire that was used in the study was translated from a CDC-ACE questionnaire [1]. The translation from English to Thai was done by the author of the study and the back translation from Thai to English was done by an independent 
linguistic professor. The back translation questions and the original questions were compared and approved by a native English speaker. The reliability score of all translated questions was 0.6 .

The Life-satisfaction question was adapted from Thai happiness indicatior-15 [19]. The questionnaire contains 15 questions. Each question had 4 options- Likert scale which ranges from 0 to 3 . The minimum score was 0 and the maximum score was 45 . The higher score means the higher life achievement and satisfaction. Each question had a factor loading greater than 4 and the reliability score of all questions were 0.7 .

\subsection{Sample Size}

Sample size was calculated using one group example. The prevalence of ACEs was based on CDC-Kaiser study that $64 \%$ of people in the study had at least one ACE. 1200 was the number of patients with psychiatric disorders aged $\geq 18$ who visited psychiatric department per month. Power 0.8 and type I error of 0.05 were used. The sample size was 270 patients.

\subsection{Statistical Analysis}

All analyses were conducted using Stata, version 15. Univariate statistics were examined for each variable exploring items missing, prevalence, and distribution. For regression analysis, a level of statistical significance at $\alpha=0.05$ was employed. The odds ratios (OR) and $95 \%$ confidence intervals (CI) were estimated from logistic regression models. Age, gender, and diagnosis were confounders that were adjusted before reporting adjusted odd ratios.

\section{Results}

271 participants completed the questionnaires. During completion of questions, one of the participants had to withdraw from the study because she had traumatic symptoms and could not finish the questions. Majority of participants were male. The mean age was 37.6 years old. The maximum age was 80 years old and the minimum age was 18 years old. Majority of participants were diagnosed as substance related disorders. $86 \%$ of participants had their education lower than a bachelor's degree. $31 \%$ of participants were married. $84.9 \%$ had their family income less than $\$ 660$ / month and $69.7 \%$ had debts.

ACEs tended to happen in females more than male (1: 0.74). Participants with substance related disorders $(45 \%)$; mood disorders $(65 \%)$; and insomnia $(66 \%)$ tended to report ACEs more than others group of participants. Participants who had ACEs tended to live alone (71\% Vs. 66\%); have less income $(87 \%$ Vs. $81 \%)$ and have more debts and consequences related to debts $(45 \%$ Vs. 31\%) than participants who had no ACE. The detail of demographic data of participants and differences of demographic data among two groups of the participants is shown in table 1 .

Table 1. The demographic data of all participants and those who did not have ACEs vs. who have at least one ACE.

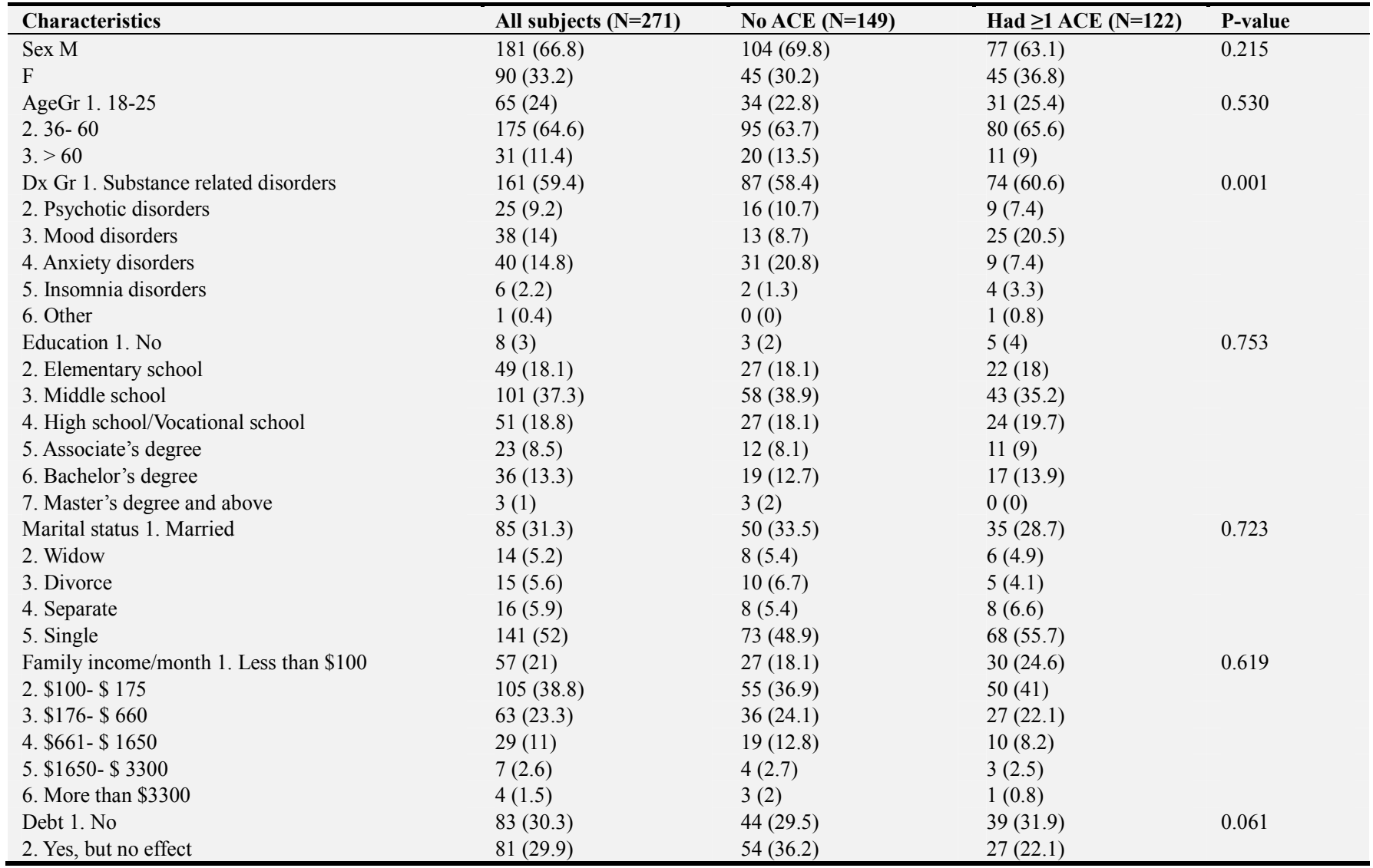




\begin{tabular}{|c|c|c|c|c|}
\hline Characteristics & All subjects $(\mathrm{N}=271)$ & No ACE $(N=149)$ & Had $\geq 1$ ACE $(N=122)$ & P-value \\
\hline 3. Yes, little effect to family & $62(23)$ & $26(17.4)$ & $36(29.5)$ & \\
\hline 4. Yes, moderate effect to family & $28(10.3)$ & $16(10.7)$ & $12(9.8)$ & \\
\hline 5. Yes, strong effect to family & $13(4.8)$ & $7(4.7)$ & $6(4.9)$ & \\
\hline
\end{tabular}

$45 \%$ of participants had at least one ACE during their age under 18 years old. The maximum events in this group of participants were 6 out of 10 events. The numbers of ACEs are shown in table 2.

Table 2. The numbers of ACEs that patients with mental illness faced before the age of 18 .

\begin{tabular}{lll}
\hline ACEs & Number & Percent \\
\hline No & 149 & 55 \\
1 & 61 & 22.5 \\
2 & 31 & 11.5 \\
3 & 15 & 5.5 \\
4 & 9 & 3.3 \\
5 & 3 & 1.1 \\
6 & 3 & 1.1 \\
\hline
\end{tabular}

The top three ACEs include feelings that they were abandoned by their families and followed by their parents divorced or separated, and were psychologically threatened by the parents. Sexual harassment and abuse was reported as the last ACE. It was reported only from 6 participants. The detail and percentage of each ACE is shown in table 3.

Table 3. Detail of ACEs and percentage of patients with mental illness who facing of each ACE.

\begin{tabular}{lll}
\hline ACEs & Number & Percent \\
\hline 1. Child psychological abuse & 33 & 12.2 \\
2. Child physical abuse & 24 & 8.9 \\
3. Child sexual abuse & 6 & 2.2 \\
4. Child emotional neglect & 55 & 20.3 \\
5. Child physical neglect & 10 & 3.7 \\
6. Parental divorced/separation & 37 & 13.7 \\
7. Mother/Stepmother had been treated violently & 8 & 3 \\
8. Household members with substance abuse & 26 & 9.6 \\
9. Household members with mental illness or suicide & 21 & 7.8 \\
10. Incarcerated household members & 18 & 6.6 \\
\hline
\end{tabular}

ACEs in patients with psychiatric disorders were associated with life satisfaction. The patients who had a higher number of ACEs had lower life-satisfaction scores. The results are shown in a graph in figure 1.

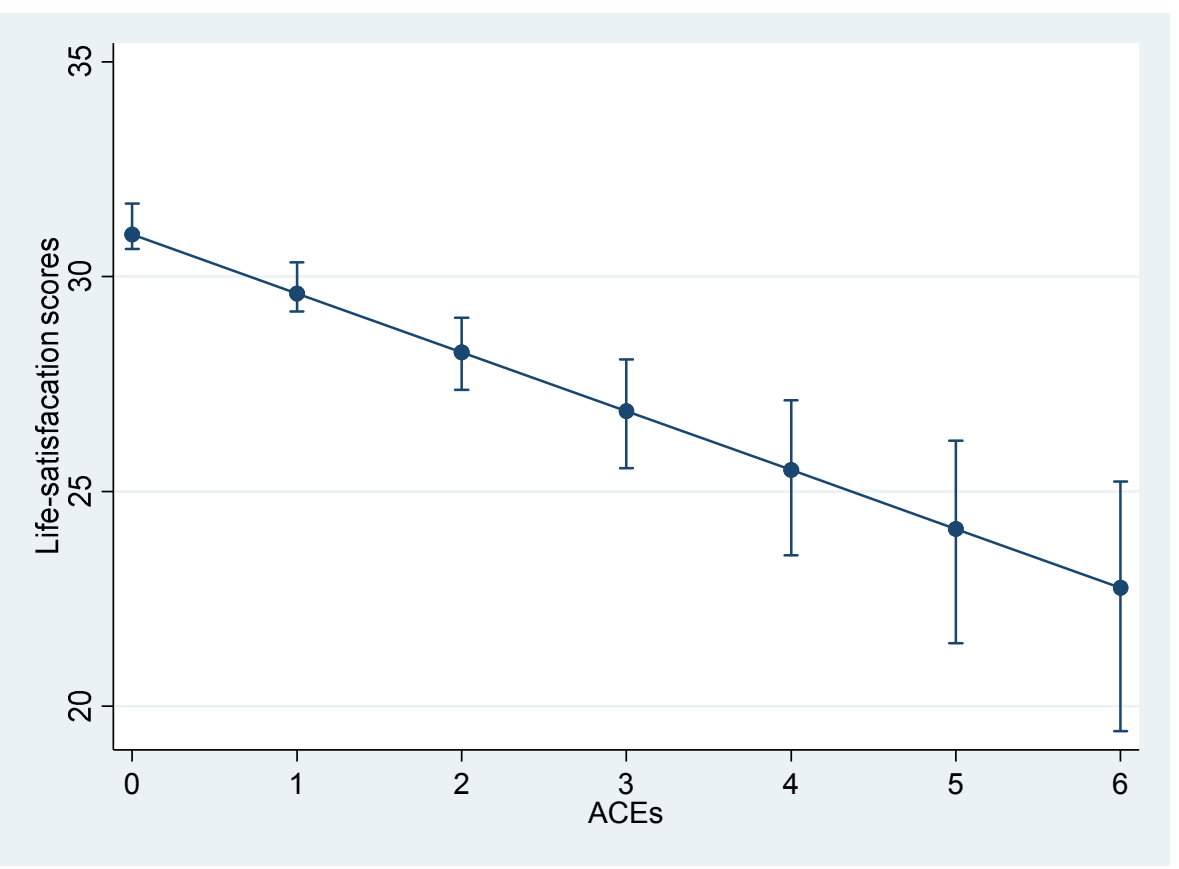

Figure 1. The graph demonstrates the association of the number of ACEs and life-satisfaction scores and adjusted prediction with $95 \%$ CI. 
Patients with psychiatric disorders who had at least one ACE tended to be less satisfied with their life, had less feelings of ease and happiness, had less feeling of safe, and love among family members, and had a difficult time to accept and handle difficulty situations. These groups of patients tended to feel bored, failure, and suffering with their lives. The detail and odd ratios of these life-satisfaction dimensions are shown in table 4.
The protective factors of ACEs were living with both father and mother, followed by living with either of them and having a family that could have been consulted and relied on during difficulties. The detail and odd ratios of the protective factors are shown in Table 4. The adjusted odd ratio was calculating by adjusting age, sex and diagnosis in the regression model.

Table 4. Comparing of life-satisfaction dimensions of patients with psychiatric disorder who had an ACE at least once and those who did not have an ACE and the unjusted odd ratio and adjusted odd ratio of protective factors of ACEs.

\begin{tabular}{|c|c|c|c|c|}
\hline Life-satisfaction Dimensions & UnAdj OR & $95 \% \mathrm{CI}$ & Adj OR & $95 \% \mathrm{CI}$ \\
\hline Satisfied with life & 0.698 & $0.585-0.833$ & 0.739 & $0.614-0.889$ \\
\hline Feeling ease and happy & 0.758 & $0.629-0.914$ & 0.779 & $0.640-0.949$ \\
\hline Feeling bored with life & 1.232 & $1.025-1.477$ & 1.242 & $1.028-1.502$ \\
\hline Feeling failure & 1.497 & $1.247-1.798$ & 1.454 & $1.206-1.751$ \\
\hline Feeling suffering in life & 1.325 & $1.099-1.597$ & 1.325 & $1.092-1.607$ \\
\hline Accepting of difficult situations & 0.774 & $0.644-0.931$ & 0.772 & $0.638-0.933$ \\
\hline Handling of difficult situations & 0.798 & $0.666-0.957$ & 0.806 & $0.668-0.972$ \\
\hline Feeling trust in a family & 0.816 & $0.683-0.974$ & 0.851 & $0.706-1.024$ \\
\hline Feeling of love and affection in the family & 0.734 & $0.610-0.883$ & 0.764 & $0.628-0.929$ \\
\hline Protective factors for ACEs & UnAdj OR & $95 \% \mathrm{CI}$ & Adj OR & $95 \% \mathrm{CI}$ \\
\hline Lived with both father and mother & 0.408 & $0.191-0.869$ & 0.383 & $0.165-0.886$ \\
\hline Lived with father or mother & 0.472 & $0.199-1.115$ & 0.376 & $0.144-0.979$ \\
\hline Family that can consult during difficulties & 0.766 & $0.452-1.298$ & 0.288 & $0.074-0.983$ \\
\hline
\end{tabular}

\section{Discussion}

In this study $45 \%$ of patients with mental illness had at least 1 ACE. This number was higher than the prevalence of the previous study of abuse by Panyayong B. in 2018 [6] that reported the prevalence of abuse was only around 5.3\% among patients with mental illness. The difference of the number might be from the definition of abuse that the previous study focused only 3 forms of abuses where as our study followed the definition of abuse of the CDC- Kaiser Permanete ACE study, that has 10 forms of abuse. This prevalence resembles previous studies that have been done among adolescents and young adults in Thailand $[5,7,8]$. However, when comparing to a study that was performed with grade 6 students [4], the prevalence of ACEs in adults was lower than the children reported. Ninety- five percent of grade 6 students reported they were abused by their parents [4]. The explanation could be the questionnaire asked the past experiences of participants at the age lower than 18 years old. Therefore there might be a recall bias among adult participants. Events which were not significant or strong enough to be memorized or traumatized may not be triggered by the questions. This hypothesis was confirmed by the sub-group analysis in our data, that participants whose age were over 60 years old tended to report less traumatic events than their younger age groups counterparts. In our sub-group analysis, around $35 \%$ of participants whose age were over 60 reported about ACES whereas the others age groups (10-year-age group divided), participants around $42-50 \%$ reported about ACEs. Female in this study had more proportion to have ACEs than male, 1:
0.74. This was according to previous studies $[3,5,9,28]$ that females had more chance to be victims than males in any age groups and females were more likely to have longer duration of having been abused. However, this finding was contrasted to some studies that reported that males had more chance to be victims than females [27]. Overall, participants in this study $(86 \%)$ had education less than a bachelor's degree and the subgroup analysis showed indifference between the group of participants who had ACEs and those who did not have. Therefore, the lower level of education of the participants in this study may primarily come from the underlying of mental illness rather than the effect of ACEs. This may imply to reviewers to cautiously interpret the previous study [1] that mentioned about limited educational success of participants with ACEs, especially those who have mental health problems, suicide, and substance use. Patients with mental illness who had ACEs tended to live alone rather than live with their partners, $(72 \%$ Vs. $67 \%)$. Overall $59 \%$ of participants in our study were married. The trend of marriage that was low among these participants might be because of the trend of the new generation life styles of Thai people [15], as the mean age of our participant was 37 and the age of 37 is considered as a gen $\mathrm{Y}$ generation. In addition this trend of living alone may be from the underlying mental illness of the patients [16] where the ACEs showed little effect but not significant in married life when comparing to previous studies that mentioned the strong effect of ACE to marital relationship. [17, 21, 22] Majority of participants had income less than $\$ 660 /$ month for both groups (with ACEs/without ACES). However, the group of participants with ACEs seemed to have more debts and problems related to debts. This assumption was confirmed by 
previous studies $[1,18,20]$ that people who had ACEs would have more socioeconomics problems comparing to general population. However, the lower income could be cautiously interpreted as it may come from the underlying mental illness of the patients. The highest prevalence of traumatic events that participants perceived was a feeling of rejection and neglect by their families. For this finding, we further did the sub group analysis of our data and found that $83 \%$ of the participants who reported this were under 45 whereas only $3 \%$ of participants whose age were over 60 reported this feeling when they were young. The explanation for this might be from the perception difference of abuse among each generation as a study by Klettke B. et al (2016) [23] which mentioned that the older generation groups (builders and baby boomers) attributed less credibility to their victim status than the younger generation groups (generation $\mathrm{Y}$ and generation $\mathrm{X}$ ). In addition, the trauma memory was usually not long lasting in people's minds if the people did not show post-traumatic stress disorder (PTSD) symptoms [24] as the memory processes are different among general people and patients with PTSD [24]. In this study group, there were no patients with PTSD. When comparing the event of emotional abuse and neglect to other list of ACEs, the other events such as physical or sexual abuse were more solid, factual, and recallable than the emotional abuse event. The second and third highest ACEs were parental divorce or separation, and physical abuse. Sexual abuse was the least reported. Two percent of participants reported about being a victim of sexual abuse. This was according to the previous studies in Thailand that the prevalence of sexual abuse was the lowest report compared to other forms of abuse. Panyayong B. al (2013) [6] found that only $0.2 \%$ of participants who had mental illness reported about sexual abuse whereas physical abuse was reported almost 25\% and being a witness of domestic violence was reported around $20 \%$ and Jirapramukpitak, T (2005) [5] reported around 5.8\% of participants had sexual abuse whereas $11.7 \%$ had physical abuse and 32\% had emotional abuse. The graded relationship was founded between the number of ACEs and the lifesatisfaction scores. Participants who had more ACEs had lower life satisfaction scores. This was confirmed by previous studies that ACEs had negative effects to life satisfaction. [1, 11-14, 18]. People who had childhood maltreatments would have less family satisfaction, less friendship satisfaction, less satisfaction with their work, less living environment satisfaction, and less self-satisfaction [29]. Nine dimensions of life-satisfaction included satisfied with life; feeling ease and happy; accepting of difficult situations; handling of difficult situations; feeling safe and love in a family; feeling bored with life; feeling failure; and feeling suffering in life were affected by ACEs. ACEs had a negative association to the first six life satisfaction dimensions, whereas the others three had positive effects. The protective factors of ACEs in this study were living with at least one of either parent or having a family that could support each other during difficulty. Although parents themselves could be perpetrators of child abuse, living with parents during childhood periods and having family that could support each other still could reduce the risk of ACEs. [2, 25,
26]

\section{Conclusion}

The prevalence of ACEs among patients with psychiatric disorders is quite common. It has been shown that ACEs or other underlying causes of psychiatric disorders, found in participants, could affect the participants' education, marriage, income, and financial debts. ACEs showed graded negative relationships with later life-satisfaction and happiness. Solutions to decrease the impacts of ACEs are to encourage parents to raise their children by themselves and to strengthen family relationships to support each other during difficulties.

\section{Limitation}

The cross-sectional nature of the study which is based on the patients with mental health illness in psychiatric clinic at Lampang Regional hospital may limit the findings.

\section{Acknowledgements}

This study was funded by Thailand mental health promotion program, department of mental health, ministry of public health, Thailand. The project number was 62-003.

\section{References}

[1] Felitti VJ, Anda RF, Nordenberg D, Edwards V, Koss PM, Marks JS. Relationship of childhood abuse and household dysfunction to many of the leading causes of death in adults. The Adverse Childhood Experiences (ACE) Study. Am J Prev Med. 1998; 14 (4): 245-58. doi: 10.1016/s07493797(98)00017-8.

[2] Panyayong B, Wacharasindhu A. Psychiatric disorders in Thai school-aged children: II Associated factors. J Med Assoc Thai 2002; 85 Suppl 1: S137-47.

[3] Finkelhor D, Hotaling G, Lewis IA, Smith C. Sexual abuse in a national survey of adult men and women: prevalence, characteristics, and risk factors. Child Abuse Negl. 1990; 14 (1): 19-28. doi: 10.1016/0145-2134(90)90077-7.

[4] Isaranurug $\mathrm{S}$, Chansatitporn $\mathrm{N}$, Auewattana $\mathrm{P}$, Wongarsa $\mathrm{C}$. Violence against children by parents. J Med Assoc Thai. 2002; 85 (8): 875-80.

[5] Jirapramukpitak T, Prince M, Harpham T. The experience of abuse and mental health in the young Thai population A preliminary survey. Soc Psychiatry Psychiatr Epidemiol. 2005; 40 (12): 955-63. doi: 10.1007/s00127-005-0983-1.

[6] Panyayong B, Tantirangsee N, Bogoian III R, Thai National Mental Health Survey Team 2013 T. Long-Term Mental Health Consequences of Child Maltreatment: A Result from Thai National Mental Health Survey 2013. J Med Assoc Thai 2018; 101: 1375-84.

[7] Htike M, Thepthein B, Chucharoen P. A web-based survey on adverse childhood experience, anxiety, depression, sexual behavior affecting methamphetamine use among adolescents in Bangkok, Thailand. Journal of Public Health and Development. 2017; 15 (3): 17-31. 
[8] Ham E, Thepthein B, Chucharoen P, Hong SA. Patterns of polysubstance use and associated factors among grade 11 students in Bangkok, Thailand. Journal of Public Health and Development, 2019; 17 (2): 1-17.

[9] Briere J, Elliott DM. Prevalence and psychological sequelae of self-reported childhood physical and sexual abuse in a general population sample of men and women. Child Abuse Negl. 2003; 27 (10): 1205-22. doi: 10.1016/j.chiabu.2003.09.008.

[10] Scheeringa MS, Zeanah CH, Myers L, Putnam FW. Predictive validity in a prospective follow-up of PTSD in preschool children. J Am Acad Child Adolesc Psychiatry. 2005; 44 (9): 899-906. doi: 10.1097/01.chi.0000169013.81536.71.

[11] Karatzias T, Chouliara Z, Power K, Brown K, Begum M, McGoldrick $\mathrm{T}$, et al. Life satisfaction in people with posttraumatic stress disorder. J Ment Health. 2013; 22 (6): 501-8. doi: $10.3109 / 09638237.2013 .819418$.

[12] Zaragoza SA. Depression, post-traumatic stress disorder, and life satisfaction in Greenlandic adults. Psyke \& Logos. 2017; 38 (1): 90-101.

[13] Mosley-Johnson E, Garacci E, Wagner N, Mendez C, Williams JS, Egede LE. Assessing the relationship between adverse childhood experiences and life satisfaction, psychological well-being, and social well-being: United States Longitudinal Cohort 1995-2014. Qual Life Res. 2019; 28 (4): 907-14. doi: 10.1007/s11136-018-2054-6.

[14] Mc Elroy S, Hevey D. Relationship between adverse early experiences, stressors, psychosocial resources and wellbeing. Child Abuse Negl. 2014; 38 (1): 65-75. doi 10.1016/j.chiabu.2013.07.017.

[15] EIC report. Report of Thai life style to be more singles. 2019 http: //www.voicetv.co.th/read/zFwdnrx2K.

[16] Jacob L, Haro JM, Koyanagi A. Relationship between living alone and common mental disorders in the 1993, 2000 and 2007 National Psychiatric Morbidity Surveys. PLoS One. 2019; 14 (5): e0215182. Published 2019 May 1. doi: 10.1371/journal.pone.0215182.

[17] Redd MJ. Adverse Childhood Experiences and Couple Relationships: Impacts on Relationship Quality and Partner Selection (Doctoral dissertation, University of Akron). 2017.

[18] Norman RE, Byambaa M, De R, Butchart A, Scott J, Vos T. The long-term health consequences of child physical abuse, emotional abuse, and neglect: a systematic review and metaanalysis. PLoS Med. 2012; 9 (11): e1001349. doi: 10.1371/journal.pmed.1001349.
[19] Mongkol A, Vongpiromsan Y, Tangseree T, Huttapanom W, Romsai P, Chutha W. The Development and Testing of Thai Mental Health Indicator Version 2007. J Psychiatr Assoc Thailand [Internet]. 2013 Apr 3 [cited 2020 Jul 2]; 54 (3): 299-316. Available from: https://he01.tcithaijo.org/index.php/JPAT/article/view/7673.

[20] Zielinski DS. Child maltreatment and adult socioeconomic well-being. Child Abuse Negl. 2009; 33 (10): 666-78. doi: 10.1016/j.chiabu.2009.09.001.

[21] Alison P, Sophie B. Child Maltreatment History and Interpersonal Problems in Adult Couple Relationships, Journal of Aggression, Maltreatment \& Trauma. 2010; 19 (2): 138-58. doi: $10.1080 / 10926770903539433$.

[22] Nguyen TP, Karney BR, Bradbury TN. Childhood abuse and later marital outcomes: Do partner characteristics moderate the association? J Fam Psychol. 2017; 31 (1): 82-92. doi: $10.1037 /$ fam0000208.

[23] Klettke B, Hallford D, Mellor D. Perceptions of credibility of sexual abuse victims across generations. Int J Law Psychiatry. 2016; 44: 91-7. doi: 10.1016/j.ijlp.2015.08.035.

[24] Wessa M, Flor H. Failure of extinction of fear responses in posttraumatic stress disorder: evidence from second-order conditioning. Am J Psychiatry. 2007; 164 (11): 1684-92. doi: 10.1176/appi.ajp.2007.07030525. PMID: 17974933.

[25] Daly, M., Wilson, M. Child abuse and other risks of not living with both parents. Ethology \& Sociobiology.1985; 6 (4): 197210. doi: 10.1016/0162-3095(85)90012-3.

[26] McLafferty M, O'Neill S, Armour C, Murphy S, Bunting B. The mediating role of various types of social networks on psychopathology following adverse childhood experiences. J Affect Disord. 2018; 238: 547-53. doi: 10.1016/j.jad.2018.06.020

[27] Thompson MP, Kingree JB, Desai S. Gender differences in long-term health consequences of physical abuse of children: data from a nationally representative survey. Am J Public Health. 2004; 94 (4): 599-604. doi: 10.2105/ajph.94.4.599.

[28] Asscher JJ, Van der Put CE, Stams GJ. Gender Differences in the Impact of Abuse and Neglect Victimization on Adolescent Offending Behavior. Journal of family violence. 2015; 30: 215-25. doi: 10.1007/s10896-014-9668-4.

[29] de Vasconcelos NM, Ribeiro M, Reis D, et al. Life satisfaction mediates the association between childhood maltreatment and depressive symptoms: a study in a sample of Brazilian adolescents. Revista Brasileira de Psiquiatria (Sao Paulo, Brazil: 1999). 2020; 42 (3): 250-257. doi: 10.1590/1516-44462019-0535. 